\title{
A dialysis accessory for attenuated total reflection infrared spectroscopy
}

\author{
Maria Krasteva, Saroj Kumar and Andreas Barth* \\ Department of Biochemistry and Biophysics, Stockholm University, Stockholm, Sweden
}

\begin{abstract}
A dialysis accessory for attenuated total reflection (ATR) infrared spectroscopy is described together with an evaluation based on known systems with well-studied infrared spectra, such as chemical oxidation and reduction of cytochrome $c$ and substrate binding to the $\mathrm{Ca}^{2+}$-ATPase. Changes in the infrared spectra of the two proteins are successfully monitored with the dialysis accessory. The accessory was developed in our laboratory for the diamond 9-reflections SensIR ATR unit. It can be used to study absorbance changes of macromolecules which are induced by low molecular weight compounds, for example the binding of substrates, inhibitors or ions to macromolecules as well as effects of $\mathrm{pH}$, ionic strength or denaturants on the structure of macromolecules. The dialysis accessory confines the macromolecule of interest to a sample compartment created between the ATR crystal and the dialysis membrane. On the other side of the dialysis membrane, a reservoir for the sample medium is created. In this way the low molecular weight compound of interest can exchange freely between the reservoir and the sample compartment via the dialysis membrane. This provides a flexible way to change sample conditions for the macromolecule of interest, allowing for example initiation of ligand binding.
\end{abstract}

Keywords: Dialysis, attenuated total reflection, ATR, vibrational spectroscopy, FTIR, ligand binding, protein

Abbreviations: $\quad$ FTIR-ATR $=$ Fourier-transformed infrared attenuated total reflection; ATP $=$ adenosine$5^{\prime}$-triphosphate.

\section{Introduction}

The use of reaction induced infrared (IR) difference spectroscopy yields valuable structural information at great detail [1-7]. A disadvantage of experiments using conventional transmission spectroscopy is the limited possibility to manipulate the sample once the IR cuvette has been closed. This has been overcome partly by applying attenuated total reflection (ATR) IR spectroscopy [8-10]. ATR spectroscopy for protein studies is mostly applied to protein films deposited on the internal reflection element. The ligands or in general any interacting compounds are exchanged on top [11-14]. Multiple experiments can be performed with different ligands on the same protein sample which saves material and time. In such experiments care must be taken so that the film is not disrupted during washing or repeated additions and withdrawals of the medium. Closing off a sample compartment by a dialysis membrane allows for precise variations of the sample conditions in a controlled way [15]. It also makes it possible to use ATR for proteins in solution. The protein is confined to a sample compartment between ATR crystal and dialysis membrane. Small molecules on the other hand can exchange freely between the reservoir and

\footnotetext{
${ }^{*}$ Corresponding author: A. Barth, Department of Biochemistry and Biophysics, The Arrhenius Laboratories for Natural Sciences, Stockholm University, S-106 91 Stockholm, Sweden. Tel.: +46 8 162452; Fax: +46 8 155597; E-mail: Andreas.Barth @ dbb.su.se.
} 
the sample compartment via the dialysis membrane. In this way one can initiate ligand binding or otherwise change sample conditions in the sample compartment. The first reported use of a dialysis setup in protein studies introduces guanosine-5'-triposphate (GTP) via diffusion through the dialysis membrane and follows the effect of GTP on a complex of the membrane protein rhodopsin with transducin [15]. Another application of dialysis ATR is the preparation of an oriented monomolecular film of porin Omp32 on a Ge internal reflecting element. Dialysis is utilized here to wash away a detergent present initially in the protein sample in order to start film formation [16]. Application of a dialysis setup for the study of metal ion binding to a soluble protein, cytochrome $c$, was described recently [17]. There is a similar dialysis set up reported by Agic et al. [18]. Both dialysis devices are built for the same ATR accessory from SensIR Technology as ours. That described in [17] uses a flow-through cell commercially available from the same company and needs $2 \mu \mathrm{l}$ of sample. That described in [18] is based on home-built cell for $4 \mu \mathrm{l}$ of sample. There is a dialysis setup commercially available from Bruker Optics which needs $50 \mu \mathrm{l}$ in the sample compartment (Anders Nilsson, Bruker Optics Scandinavia AB, private communication). Here we report our setup for the diamond 9 reflections SensIR FTIR-ATR accessory with a sample compartment of $3 \mu \mathrm{l}$.

\section{Materials and methods}

Circles of $8 \mathrm{~mm}$ in diameter, cut out of cellulose sheets CelluSep F3 of MWCO 12000-14000 dialysis membranes (Orange Scientific, Belgium), were used for the dialysis accessory.

Horse heart cytochrome $c$ (cyt $c$ ) was purchased from Sigma and used without further purification. The cyt $c$ samples were prepared in $100 \mathrm{mM}$ phosphate buffer $\mathrm{pH} 6.8$, containing $100 \mathrm{mM} \mathrm{KCl}$. The final concentration of cyt $c$ was $8 \mathrm{mM}$. Solutions of potassium ferricynide and sodium dithionate for cyt $c$ oxidation and reduction were both $200 \mathrm{mM}$.

The FTIR spectra of cyt $c$ were recorded at $4 \mathrm{~cm}^{-1}$ resolution on a Bruker Vertex 70 FTIR spectrometer equipped with an $\mathrm{MCT}$ detector. The experiments were performed at room temperature. Cyt $c$ was equilibrated by continuous diffusion of medium (phosphate buffer with $\mathrm{KCl}$ ) across the dialysis membrane. After the absorption spectrum of the sample became stable, a 500 scan single beam spectrum (background spectrum) was recorded. Then $4 \mu \mathrm{l}$ of potassium ferricynide $(200 \mathrm{mM})$ were added to the reservoir after which an acquisition of repeated spectra in the absorption mode, 150 scans each, was initiated. Under these conditions, oxidation of cyt $c$ was first detected after 20 seconds and monitored until it was complete after 5 minutes. The system was brought back to the initial state by exchanging three to four times the sample medium in the reservoir with fresh one in 5-10 minute intervals. Cyt $c$ was reduced by adding $2 \mu \mathrm{l}$ sodium dithionate $(200 \mathrm{mM})$ to the reservoir. The reaction was also first detected 20 seconds later and a completion was reached 5 minutes later. Typically, one oxidation-reduction cycle was carried out for 30-40 min.

$\mathrm{Ca}^{2+}$-ATPase was measured in a medium of $100 \mathrm{mM}$ TRIS pH 7 with $10 \mu \mathrm{M} \mathrm{MgCl}_{2}, 100 \mu \mathrm{M} \mathrm{CaCl}_{2}$ and $100 \mathrm{mM} \mathrm{KCl}$. A stock solution of $\mathrm{Ca}^{2+}$-ATPase sarcoplasmic reticulum vesicle suspension, prepared as given in [19] ( $80 \mu \mathrm{l})$, was dialyzed for 1 hour in 10 times diluted medium, then concentrated to a final concentration of around $150 \mathrm{mg} / \mathrm{ml}$ by adding aliquots of $20 \mu \mathrm{l}$ on the ATR crystal and drying with a flow of $\mathrm{N}_{2}$. The last $8 \mu \mathrm{l}$ were used for closing the dialysis setup. The FTIR spectra of $\mathrm{Ca}^{2+}$-ATPase were recorded at $4 \mathrm{~cm}^{-1}$ resolution on a Bruker IFS 66/S spectrometer equipped with an MCT detector. The temperature for these measurements was $5^{\circ} \mathrm{C}$. Single beam background spectra were taken with 1000 scans. Then the $\mathrm{Ca}^{2+}$-ATPase substrate adenosine-5'-triphosphate (ATP), disodium salt, was added as $2 \mu \mathrm{l}$ of $60 \mathrm{mM}$ solution to the reservoir and several spectra recorded with 300 scans each. 


\section{The dialysis accessory}

\subsection{Description}

The sample is placed between the total reflection surface of the ATR reflection element and the dialysis membrane (Fig. 1). The membrane is fixed by an O-ring, which snaps into a groove on the bottom conical part of the ligand reservoir. The reservoir is made of delrin - a polyacetal resin polyoxymethylene. The reservoir is let slide down in a cylindrical opening of a thermostating block, which can be assembled and secured tightly to the ATR bench. It sits on the horizontal plane of the metal block that covers the ATR unit. The height of the dialysis membrane above the internal reflection element is determined by the height of the bottom conical part of the reservoir. The ligand reservoir is pressed down by a screw cap which screws onto a thread on the thermostating block. The O-ring makes a tight contact with the walls of the conical dip in the metal block of the ATR unit. Thus a tight closure of the sample compartment is achieved while no pressure is exerted on the total reflection surface of the ATR reflection element. The latter point is crucial since the diamond ATR crystal, although inert and therefore not demanding in terms of the nature of the liquid samples measured, is on the other hand very sensitive to pressure since it is a thin diamond plate supported only on the periphery and not across the entire surface. The setup reported by Gourion-Arsiquaud et al. [17] uses pressure on the periphery which might impose the risk of breaking the crystal.

\subsection{Operation of the dialysis accessory}

The dialysis membrane is first soaked for 1-2 min in double distilled water and transferred without drying to the end of the conical opening of the reservoir. The membrane is attached to the reservoir by snapping a wet O-ring into the groove on the reservoir. The reservoir is then filled with the sample medium. A drop of $4 \mu \mathrm{l}$ from our protein sample solution is placed on the total reflection surface of the ATR crystal. Another $4 \mu \mathrm{l}$ of the sample are deposited as a hanging drop on the dialysis membrane at the bottom of the reservoir. The reservoir slides down the opening in the thermostating block and the setup is closed with the screw cap on top. The solution in the reservoir is stirred constantly with a small mechanical stirrer attached at the top. Initially we were closing with a total of $8 \mu \mathrm{l}$ sample volume and the controls we did and show in the paper are with $8 \mu \mathrm{l}$ but later we found out that the setup can work with as little as $3 \mu \mathrm{l}$.

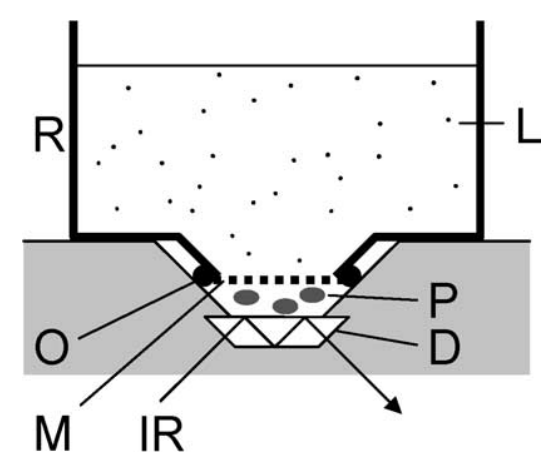

Fig. 1. Dialysis accessory for the diamond 9 reflections SensIR ATR unit. R: reservoir, O: O-ring, M: dialysis membrane, IR: infrared beam, D: diamond plate, P: protein (as an example for a macromolecule), L: ligand (as an example for the low molecular weight compound). 


\subsection{Equilibrating the system before the beginning of the experiments}

Two to three hours after assembling the unit experiments can be started. The reason for the waiting time is that the absorption of the sample changes due to processes within the protein sample. The smaller the concentration of the sample is, the shorter it takes to obtain a stable absorption. Even if dialyzed protein is transferred to the ATR crystal, and the reservoir contains the dialysis buffer, it takes time before the absorption is stable. In our experiments, the ligands were added as solutions with the same concentrations of buffer and salts as the sample medium in the reservoir. A second possibility is to exchange the medium in the reservoir with a fresh one that has the same composition as before and in addition contains the ligand of interest (results not shown).

\section{Results and discussion}

A first test of our dialysis accessory was whether low molecular weight compounds added to the reservoir would arrive in the sample compartment. Multiple additions of for example ATP to the reservoir revealed that signals of added compounds can be observed within $10 \mathrm{~s}$ after the addition if the ligand is at high concentrations $\left(10^{-2} \mathrm{M}\right.$ range final concentration). At lower concentrations $\left(10^{-4}-10^{-5} \mathrm{M}\right.$ range) the times lengthens 10 -fold to 100 seconds. In these measurements the arrival of the low molecular weight compound in the sample compartment was detected directly via the infrared absorption of the compound. This is generally no longer possible if the concentration is in the $\mu \mathrm{M}$ range. Recent measurements on a soluble protein and its inhibitor showed that at these concentrations ( $10^{-6} \mathrm{M}$ range), arrival of the ligand in the sample compartment can be detected indirectly from difference signals arising from changes in the protein bands upon protein-ligand interaction and they take longer times to be observed (10-40 minutes, results not shown).

The difference spectra corresponding to reduction and reoxidation of cyt $c$ are presented in Fig. 2. The two spectra are almost exact mirror images of each other. Both spectra are found to be fully reproducible in a large number of cycles of a single sample and display all the spectral features found in previous infrared studies of redox induced changes in the infrared absorption of cyt $c$ [17,20-22]. In the earlier work, reduction and oxidation of cyt $c$ was induced by electrochemical means. These reports also contain detailed analyses of the spectra with the current state of the band assignments. The agreement with previous results shows that our dialysis accessory can be used to obtain high quality spectra of a protein reaction. We note that the observed absorbance changes are smaller than $0.1 \%$ of the typical absorbance of an aqueous protein sample in transmission experiments.

Difference spectra of the $\mathrm{Ca}^{2+}$-ATPase revealing the changes in protein absorption after the addition of ATP are presented in Fig. 3. Spectrum A was recorded $80 \mathrm{~s}$ after the addition and represents changes in the absorption that are not related to ATP binding to the ATPase. The two largest bands in this spectrum at 1531 and $1067 \mathrm{~cm}^{-1}$ coincide with bands of the buffer tris. These changes persist throughout the measurements and can be seen together with the changes in the protein observed at later times (Fig. 3B, 120 seconds after the addition). Figure 3C presents the spectrum in Fig. 3B corrected for the buffer absorbance change seen in Fig. 3A. The observed signals agree with spectra taken in the transmission mode, considered indicative of nucleotide binding to the $\mathrm{Ca}^{2+}$-ATPase $[23,24]$. They were obtained using caged compounds, which photolytically release the compound of interest from an biologically inactive photosensitive precurser. While this approach is powerful, it requires the synthesis of an appropriate caged compound which is time-consuming and might be difficult to achieve. The work presented here shows that dialysis coupled ATR spectroscopy can be used as an alternative to monitor ligand binding to proteins. 


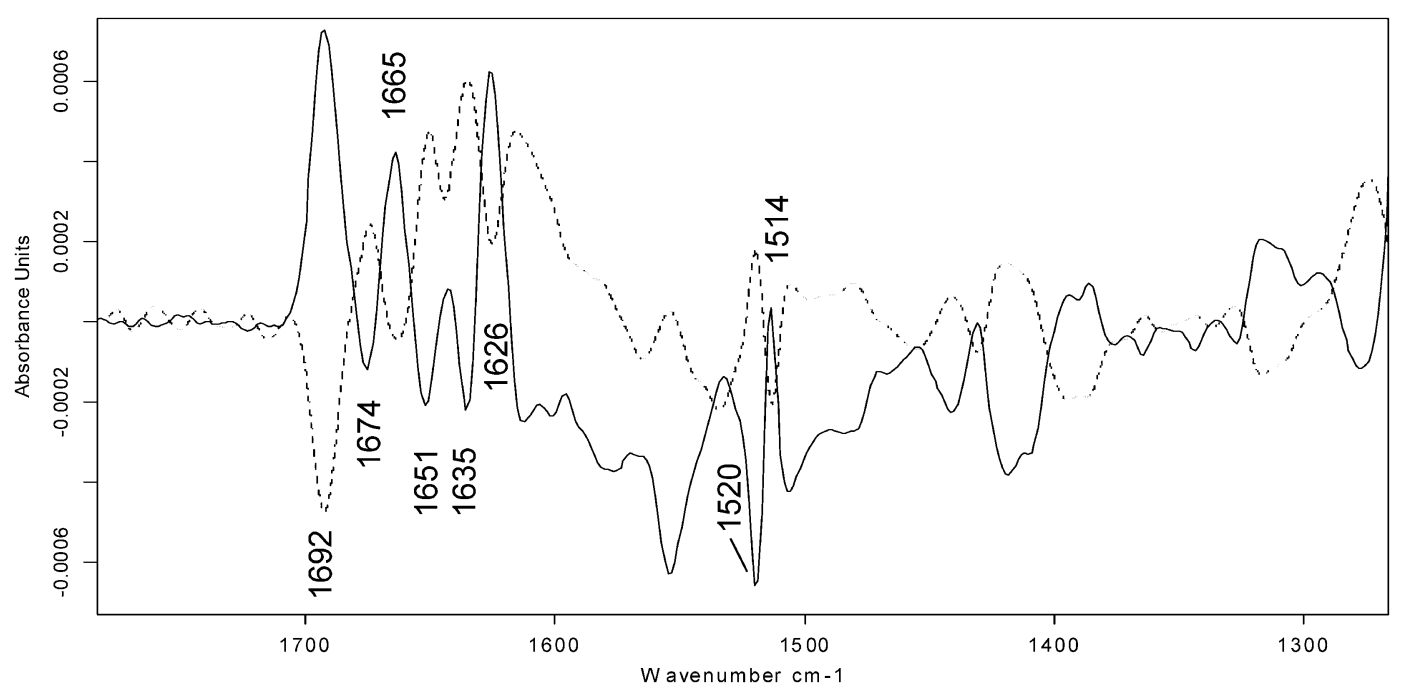

Fig. 2. Infrared difference spectra of reduced minus oxidized cyt $c$ (solid line) and of oxidized minus reduced cyt $c$ (dotted line). Reduced minus oxidized cyt $c$ was obtained upon addition of reducing agent sodium dithionate $(2 \mu 1,200 \mathrm{mM})$ to the reservoir of the ATR accessory (final concentration $1 \times 10^{-4} \mathrm{M}$ ). Concentrations in the sample compartment were $8 \mathrm{mM}$ cyt $c$, $100 \mathrm{mM}$ phosphate buffer, $\mathrm{pH} 6.8,100 \mathrm{mM} \mathrm{KCl}$. Oxidized minus reduced cyt $c$ was obtained upon addition of oxidizing agent potassium ferricynide $\left(4 \mu \mathrm{l}, 200 \mathrm{mM}\right.$, final concentration $\left.2 \times 10^{-4} \mathrm{M}\right)$.

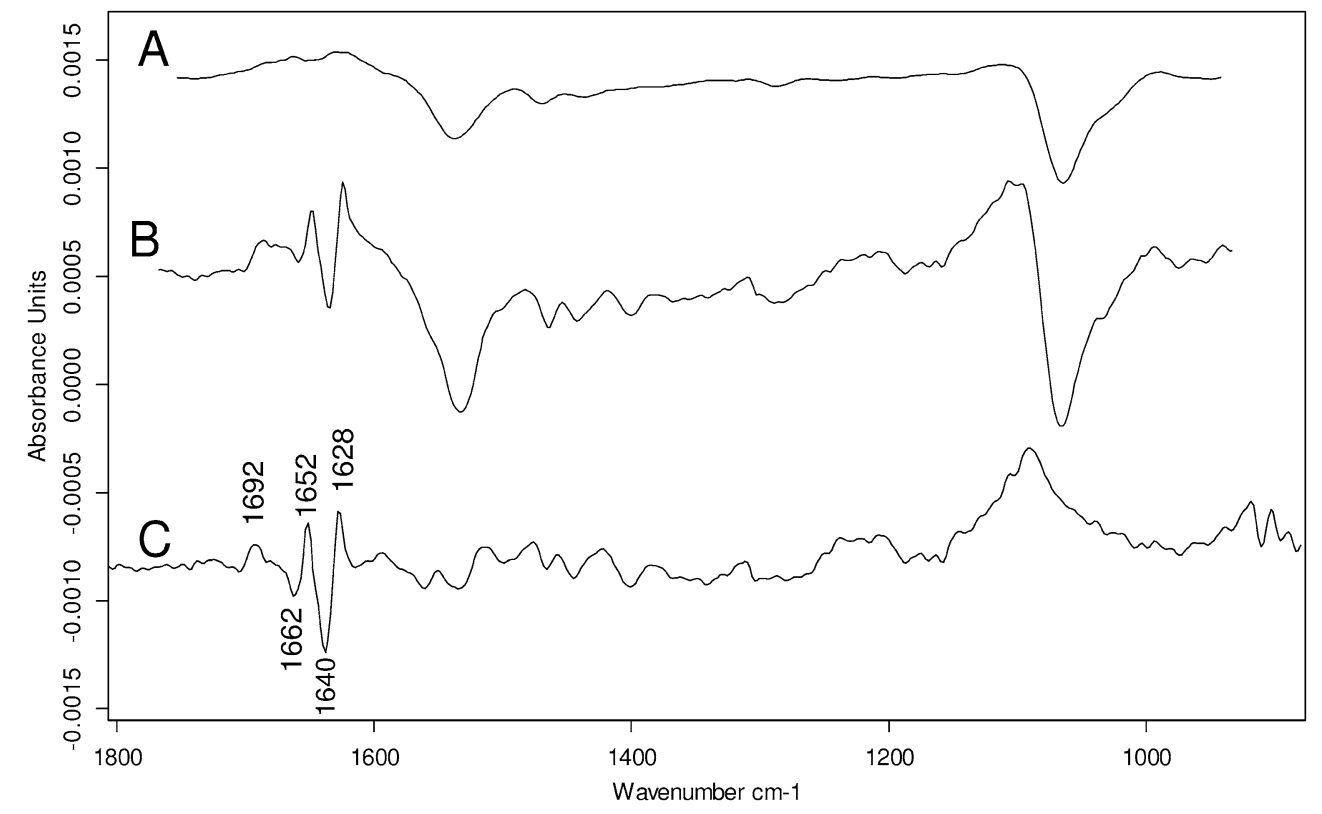

Fig. 3. Infrared difference spectra of $\mathrm{Ca}^{2+}$-ATPase representing changes upon addition of ATP $(2 \mu \mathrm{l}, 60 \mathrm{mM}$, final concentration $3 \times 10^{-5} \mathrm{M}$ ) via perfusion through the dialysis membrane of the dialysis accessory. A: observed spectral changes $80 \mathrm{~s}$ after the addition; $\mathrm{B}: 120 \mathrm{~s}$ after the addition; $\mathrm{C}: \mathrm{Ca}^{2+}$-ATPase changes $120 \mathrm{~s}$ after the addition (spectrum $\mathrm{B}$ ) corrected for the baseline changes seen in spectrum A. 


\section{Conclusions}

While reaction-induced infrared difference spectroscopy is a highly sensitive and highly informative method, the difficulty of inducing reactions or perturbations has been a major bottleneck for its general usability. The use of dialysis accessories to ATR units is becoming to overcome this bottleneck since it provides a general method to study perturbations of macromolecules with infrared spectroscopy. This work describes our dialysis accessory for the 9 reflections SensIR ATR unit and we showed that high quality spectra can be obtained with it using two examples - chemical oxidation and reduction of cyt $c$ and substrate binding to the $\mathrm{Ca}^{2+}$-ATPase. As dialysis coupled ATR infrared spectroscopy makes infrared spectroscopy a more universal method, in particular for studying the binding of small molecules to macromolecules, we expect infrared spectroscopy to expand to new fields, for example to that of drug development.

\section{Acknowledgements}

The authors are grateful to W. Hasselbach (Max-Planck-Institut, Heidelberg) for the gift of $\mathrm{Ca}^{2+}$ ATPase. This work was supported by Vetenskapsrådet projektbidrag 621-2002-5884, and Knut och Alice Wallenbergs Stiftelse bidrag 2002.0115. MK acknowledges a post-doc grant from Wenner-Grenska Samfundet.

\section{References}

[1] C. Zscherp and A. Barth, Biochemistry 40 (2001), 1875-1882.

[2] S. Kim and B.A. Barry, J. Phys. Chem. 105 (2001), 4072-4083.

[3] K. Gerwert, Biol. Chem. 380 (1999), 931-935.

[4] C. Jung, J. Molec. Recognit. 13 (2000), 325-351.

[5] W. Mäntele, Trends in Biochemical Sciences 18 (1993), 197-202.

[6] C.W. Warton, Nat. Prod. Rep. 17 (2000), 447-453.

[7] R. Vogel and F. Siebert, Curr. Opin. Chem. Biol. 4 (2000), 518-523.

[8] N.J. Harrick, in: Internal Reflection Spectroscopy, Harrick Scientific Corp., Ossining, NY, 1979.

[9] U.P. Fringeli, in: Internal Reflection Spectroscopy. Theory and applications, Quantum Chemical Corp., Morris, IL, 1992.

[10] E. Goormaghtigh, V. Raussens and J.-M. Ruysschaert, Biochim. Biophys. Acta 1422 (1999), 105-185.

[11] J.E. Baenzinger and J.P. Chew, Biochemistry 36 (1997), 3617-3624.

[12] U.P. Fringeli, H.-J. Apell, M. Fringeli and P. Läuger, Biochim. Biophys. Acta 984 (1989), 301-312.

[13] F. Scheirlinckx, V. Raussens, J.-M. Ruysschaert and E. Goormaghtigh, Biochem. J. 382 (2004), 121-129.

[14] R.M. Nyquist, D. Heitbrink, C. Bolwien, T.A. Wells, R.B. Gennis and J. Heberle, FEBS Lett. 505 (2001), 63-67.

[15] K. Fahmi, Biophys. J. 75 (1998), 1306-1318.

[16] M. Schwarzott, H. Engelhardt, T. Kluehspies, D. Baurecht, D. Naumann and U.P. Fringeli, Langmuir 19 (2003), $7451-$ 7459.

[17] S. Gourion-Arsiquaud, S. Chevance, P. Bouyer, L. Garnier, J.-L. Montillet, A. Bondon and C. Berthomieu, Biochemistry 44 (2005), 8652-8663.

[18] E. Agic, O. Klein and W. Mäntele, in: Book of Abstracts, 10th European Conference on the Spectroscopy of Biological Molecules, Aug. 30-Sept. 4 (2003), Szeged, Hungary.

[19] L. De Meis and W. Hasselbach, J. Biol. Chem. 246 (1971), 4759-4763.

[20] D. Moss, E. Nabedryk, J. Breton and W. Mäntele, Eur. J. Biochem. 187 (1990), 565-572.

[21] D.D. Schlereth and W. Mäntele, Biochemistry 32 (1993), 1118-1126.

[22] K. Ataka and J. Heberle, JACS 126 (2004), 9445-9457.

[23] A. Barth, F. von Germar, W. Kreutz and W. Mäntele, J. Biol. Chem. 271 (1996), 30637-30646.

[24] F. von Germar, A. Barth and W. Mäntele, Biophys. J. 78 (2000), 1531-1540. 


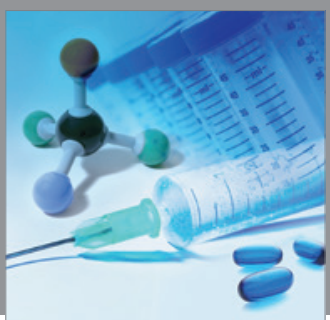

International Journal of

Medicinal Chemistry

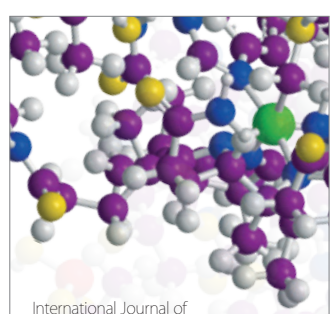

Carbohydrate Chemistry

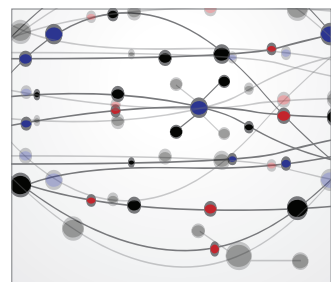

The Scientific World Journal
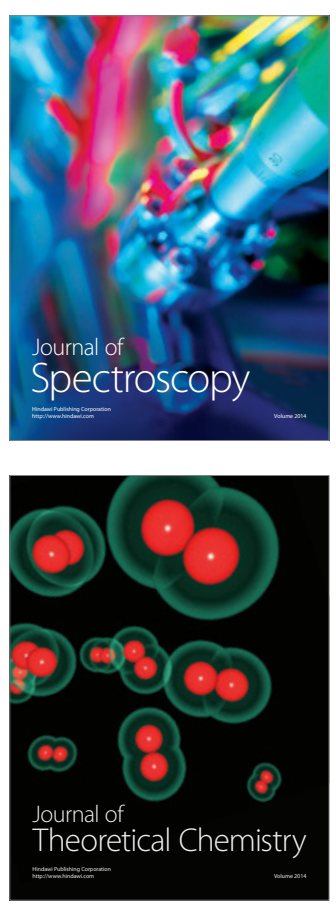
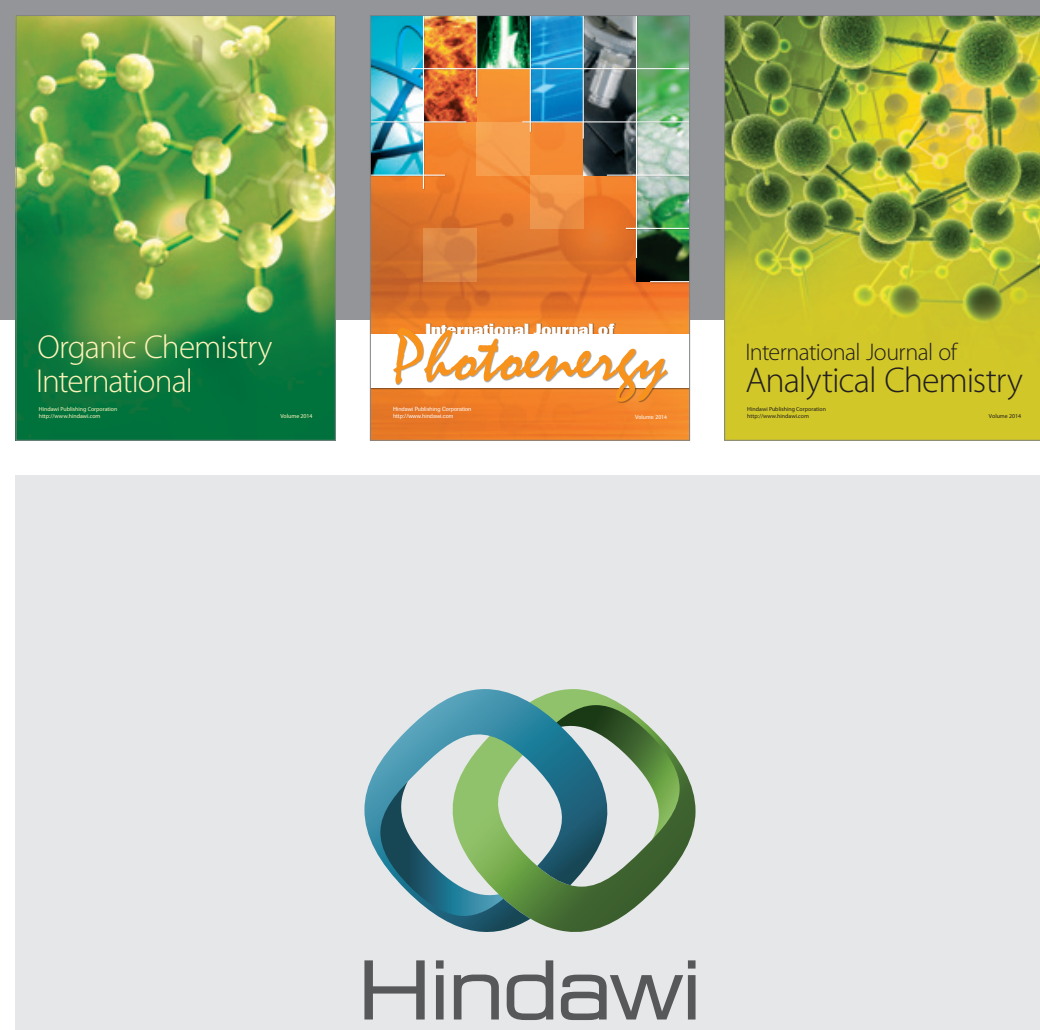

Submit your manuscripts at

http://www.hindawi.com
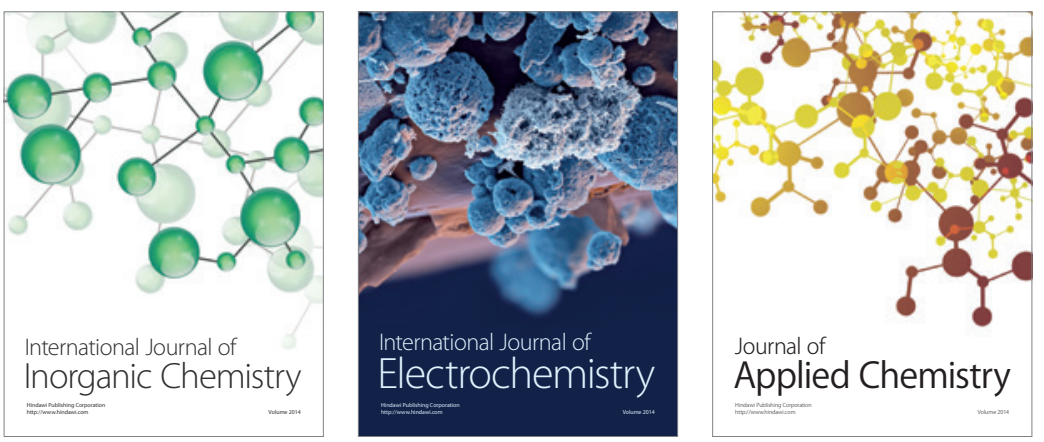

Journal of

Applied Chemistry
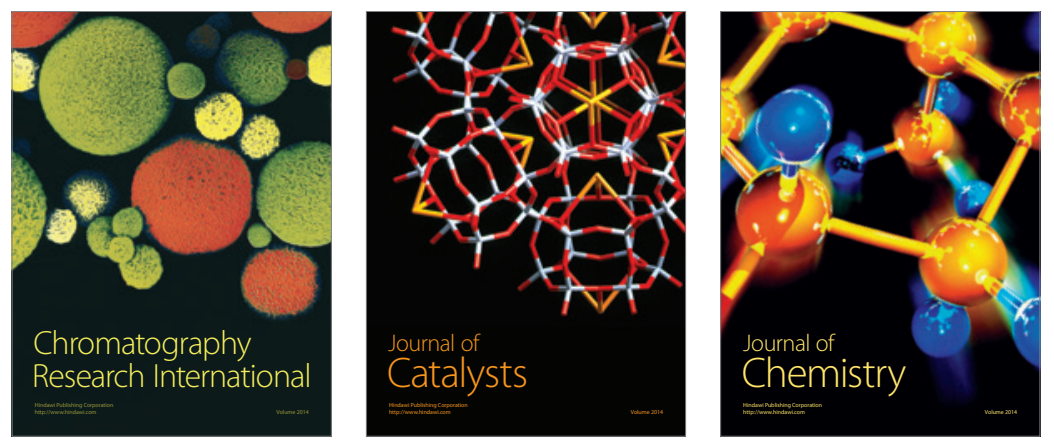
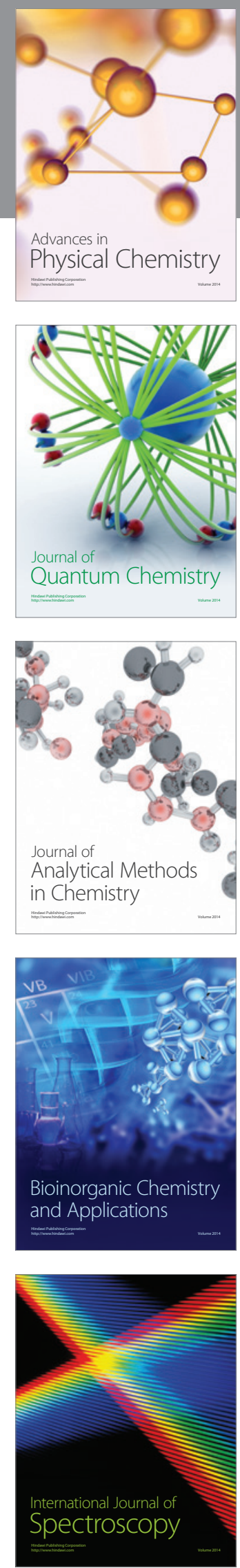